every now and then; tongue foul, and very dark; the ulcerations on the abdomen have a bad appearance; no stool, except after lavement. An emollient enema.

18. The patient has cried less during the last two days; skin moderately warm; pulse small, 108 ; goes to stool without enema; the evacuations are not liquid; she shows the tongue well, and is less deaf; the aspect of the ulcers is improved.

19. No answers to any question; gives no sign of intelligence, except by putting out the tongue when asked; deglutition difficult; slight cough without expectoration; respiration not accelerated; the chest sounds well on percussion; the cellular tissue of the neck is becoming emphysematous.

20. Same state; emphysema considerably augmented.

22. No great improvement or change has taken place; she is less deaf, and now answers a few questions; tongue moist; has passed a few fluid stools; pulse 108; ulcers on abdomen healed; the emphysema persists.

24. Pulse very irregular; she now answers pretty well; no diarrhœa; the emphysema has extended considerably.

26. No diarrhœa; she now answers all questions distinctly; pulse 92 ; slin cool; ordered to have some nourishing soup.

30. Is excessively emaciated and weak; all the sores have healed; no deafness or headache; intellect quite clear; tongue moist and clean; no pain in abdomen; no diarrhœa; pulse 72, On the 1st of April she was allowed to leave her bed for the first time, and was discharged cured on the 1st of May.

\section{PROVINCIAL HOSPITALS.}

TO THE EDITORS OF THE PROVINCIAL MEDICAL AND SURGICAL JOURNAL.

GentLemen,-In a late number of your journal, I observe some excellent remarks on provincial schools of medicine. As provincial hospitals are very properly connected with these schools, may I be permitted to direct your attention to their government and medical polity. My object in addressing you is chiefly for the purpose of eliciting your opinion, and the opinions of those who have devoted their attention to the subject of hospital attendance, relative to the best plan of providing medical and surgical attendance for county hospitals.

A county infirmary has been lately erected at Carlisle, which contains accommodation for fifty-two beds ; and some difficulty has occurred in framing rules for the medical officers, and in making the medical appointments. Only twelve beds are yet fitted up, and the annual subscriptions at present are not adequate to the maintenance of a greater number. According to the rules that have been adopted by the governors, the number of medical officers is limited to four, viz. two physicians and two surgeons, and the following are the rules respecting their qualifications. "No one shall be eligible to the office of physician to the infirmary, unless he be a medical graduate of the University of Oxford, Cambridge, Edinburgh, Dublin, Glasgow, or London; nor shall any person be eligible who practises, or is connected in partnership with any one who practises surgery, pharmacy, or midwifery. No person shall be eligible to the office of surgeon to the infirmary, unless he be a member of the College of Surgeons of London, Dublin, Edinburgh, or Glasgow."' These rules, I believe, are similar to the rules of other provincial infirmaries. Out of seventeen medical gentlemen now resident in Carlisle, fifteen object to these rules, and decline offering themselves as candidates to fill the vacant offices, as you will find by the following memorial and address, which they have presented to the governors. The vacant offices are consequently not filled up.

[It is unnecessary to print the memorial and address in full; we, therefore, insert an abstract of them here. The memorialists urge, that it would be preferable to merge all distinctions of rank or mode of practice, and throw open the elections to all who are qualified to practise either medicine or surgery by the respective universities or colleges. They also think, that instead of limiting the number of medical officers to four, the interest of the institution would be better consulted by admitting the largest possible number of legally-qualified practitioners, of whom a certain number at a time might act in annual or half-yearly rotation. The same principles are more fully developed in an address to the governors of the infirmary, adopted at a meeting of the resident practitioners of Carlisle, held on September 20, 1841.

The memorial and address are signed by the following gentlemen :-William Jackson, M.D.; Thomas Elliot, surgeon; Richard James, M.D., \&c. ; James Marrs, surgeon; R. Atkinson, M.D., \&c. \&c.; Edward Bowman, surgeon; R. Oliver, Licentiate Royal College of Physicians, London; Peter Linton, surgeon; William Elliot, M.D.; Francis W. Kerr, surgeon; John Mortimer, surgeon; Joseph Cartmell, M.D.; John Hodgson, surgeon; William Dalton, M.D.; Wm. Nicholson, surgeon.-EDs.]

The plan of electing the largest possible number of legally-qualified practitioners, of whom a certain proportion at a time shall act in annual or half-yearly rotation, is, I think, perfectly new, and would not be desirable or advantageous to the institution. It is a very different plan, as far as I have been able to learn, from what is adopted in other county infirmaries, and just the reverse of what has been recommended by Dr. Clark and Dr. Walker, who have written on the subject.

"With regard to the number of physicians and surgeons," says Dr. Walker, " necessary to conduct the business of an infirmary, it seems to be conceded by the most competent judges, that the interest of hospital patients is best consulted by having no more medical attendants than are sufficient for the care of the complaints. Every supernumerary officer, by dividing, lessens the responsibility of the rest. In county infirmaries, too many medical officers are frequently appointed, from which cause the business becomes so much divided, as to afford a very limited field of experience. This applies peculiarly to the operative part of surgery, where dexterity, expertness, and skill are only to be acquired by almost constant practice."* Dr. Clark, who has expressed himself very deciciedly upon this subject, considers

* On the Rules and Regulations of English Hospitals. By J. 5. Walker, M.D.-Hidlnd Medical Reporter. 
that in an infirmary, capable of accommodating, at one time, as many as 150 in-patients, two ordinary physicians and two surgeons are enough to transact the business ; but he recommends, where the number of beds is so considerable, the appointment of a similar number of assistant medical officers, to supply the places of the ordinary physicians and surgeons, in cases of absence, death, or resignation.*

In a table of provincial infirmaries, drawn up by Dr. Walker, and in some hospital reports now before me, I find that the following hospitals have each two physicians and two surgeons, viz. York, Lancaster, Gloucester, Chichester, Durham, Dumfries, Hereford, and Chester. The following have each three physicians and three surgeons, Hull, Liverpool, Leeds, Norfolk, Sheffield, Worcester, and Reading. Derby, Northampton, Huddersfield, and Stafford have each two physicians and three surgeons. The Glasgow and Lincoln infirmaries have each two physicians and four surgeons. The Bedford infirmary has one physician and two surgeons. You will observe that six medical graduates have signed the memorial and address. 'These gentlemen object particularly to the latter part of the rule, which relates to the qualification of physicians. They take general practice, and say it is extremely hard and very improper, that, on account of their being practitioners, they should be disqualified by the rule from being eligible to be physicians to the infirmary. One gentleman is a licentiate of the College of Physicians of London, and several of them, I understand, are licentiates of the College of Surgeons of Edinburgh. In order to conciliate these gentlemen, and render them all eligible to fill the offices of physicians and surgeons, the governors have altered the aloove rules, and have adopted the following: "'That no person shall be eligible to the office of physician to the infirmary, unless he be a graduate of a British or Irish university, or licentiate of the College of Physicians of London. 'That no person shall be eligible to the office of surgeon to the infirmary, unless he be a member or licentiate of the College of Surgeons of London, Dublin, Edinburgh, or Glasgow." Although all the gentlemen who signed the memorial and address are now made eligible, not one of them, I am informed, will take office.

The subject of hospital attendance is of great importance, and not only deserves the attention of the editors of the Provincial Medical and Surgical Journal, but also the best consideration of the members of the association. I therefore hope you will insert this communication in your journal, and also take an early opportunity of favouring your readers with an editorial article on the subject. It would likewise be interesting and useful to many of your readers to be informed of the number of beds a provincial hospital must contain to be recognised by the examining boards in London, and if any, and what particular rules and regulations are required by these boards.

$$
\text { Yours, \&c., }
$$
Cumbriensis.

Carlisle, September 24, 1841.

$$
\text { - Collection of Papers. By J. Clark, M.D. }
$$

\section{HERNIA REDUCED BY ICE.}

TO THE EDITORS OF THE PROVINCIAL MEDICAL AND SURGICAL JOURNAL.

Gentegmen,-As every contribution tending to diminish the necessity of operations in surgery $I$ deem to be of importance, perhaps the following facts may be regarded of that nature; and although the practice referred to is well known, yet I doubt whether it be so frequently resorted to as may be desirable, before the last and often fatal practice be adopted,-I mean the operation for strangulated hernia.

Cases of hernia, we all know, are not frequent in private practice, and when they do occur, the surgeon soon gets anxious for the fate of his patient, and very desirous to ward off an operation, which, even in the most skilful hands, is a very serious and dangerous affair. It has unhappily fallen to my lot to see the operation performed, and the patient die within four hours after, and die most evidently from the operation itself, and not from the previous state of the intestine. It is therefore with very great pleasure I can state the complete success in two cases of the application of ice to the tumor. In one of the cases, a female had inguinal hernia of the right side; her symptoms were severe, she had vomiting, pain on pressure, and a good deal of general anxiety. 'The usual remedies were adopted, and as the symptoms were urgent, it was resolved to operate; but, at the suggestion of a brother practitioner, I had a bladder of ice kept for some hours over the tumor; the result was a quick relief from pain, and a very slight manipulation sufficed to return the intestine. In the other case, the patient was a strong seafaring man, who had suffered the usual symptoms of strangulated inguinal hernia for twenty-four hours before I saw him; after bleeding, I attempted reduction by the taxis, but was compelled to desist from the pain it produced. Ice was now applied, and in three hours afterwards the man reduced the tumor himself, without any difficulty. 'The immediate effect of this application in such cases is very satisfactory to the patient; the tension of the part is removed, and consequently the pain: then follows the diminution of bulk by the contraction produced, which again seems happily to end in the return of the intestine.

Should the foregoing statement lead surgeons to the more general trial of this remedy before proceeding to operate, I shall feel much satisfaction.

Your obedient servant,

Edward Humpage.

Bristol, Sept. 27, 1841.

\section{GRATUITOUS SERVICES OF MEDICAL MEN.}

TO THE EDITORS OF THE FROVINCIAL MEDICAL AND SURGICAL JOURNAL.

Gentumane, - I send you the Liverpool Standard, which contains a letter from me, signed "Medicus," for the purpose of reprinting it in the Provincial Medical and Surgical Journal, if you think the subject of sufficient interest to your readers. It may, perhaps, appear strange that I should hazard such sentiments as those of remu- 